\title{
NOTE ON LEFT SERIAL ALGEBRAS
}

\author{
$\mathrm{By}$
}

\author{
Manabu HARADA
}

(Dedicated to the memory of Professor Akira HATTORI)

Let $R$ be a left and right artinian ring with identity. We have studied the condition $(*, n)$ : every maximal submodule of direct sum of arbitrary $n R$-hollow modules is also a direct sum of hollow modules [1].

We shall study, in this short note, some left serial rings satisfying $(*, 1)$ for right $R$ module, and give a characterization of such a left serial algebra with $J^{4}=0$.

\section{§1. Algebras of right local type}

Let $R$ be a left and right artinian ring with identity. We assume that every $R$-module is a unitary right $R$-module and we denote the Jacobson radical and the socle of an $R$-module $M$ by $J(M)$ and Soc $(M)$, respectively. We put $J=J(R)$, and $|M|$ means the length of a composition series of $M$. Following $H$. Tachikawa [5], $R$ is called a ring of right local type, if every finitely generated right $R$-module is a direct sum of local (hollow) modules. We are sometimes interested in an algebra $R$ over a field $K$ with the following condition:

(A) $e R e / e J e=e K+e J e$ for each primitive idempotent $e$, (Condition II" in [1], e.g., $K$ is an algebraically closed field).

$T$. Sumioka found the following remarkable result for a left serial ring $R$ [4]:

LEMMA 1. ([4], Corollary 4.2). Let $R$ be a left serial ring, then eJ $^{i}$ is a direct sum of hollow modules as right $R$-modules for any $i$.

On the other hand, if $R$ satisfies $(*, 1)$, then $e J^{i}$ has the same structure from the definition (cf. [3], §1). Further we obtained

LEMMA 2. ([3], Theorem 4). Let $R$ be a right artinian ring. Then $R$ satisfies $(*, 1)$ for any hollow module if and only if the following two conditions are fulfiled:

1) $e J=\sum_{i=1}^{n(e)} \oplus A_{i}$, where $e$ is any primitive idempotent in $R$ and the $A_{i}$ are hollow.

2) Let $C_{i} \supset D_{i}$ be two submodules of $A_{i}$ such that $C_{i} / D_{i}$ is simple. If $f: C_{i} / D_{i} \approx C_{j} / D_{j}$ for $i \neq j, f$ or $f^{-1}$ is extendible to an element in $\operatorname{Hom}_{R}\left(A_{i} / D_{i}, A_{j} / D_{j}\right)$ or $\operatorname{Hom}_{R}\left(A_{j} / D_{j}\right.$, $\left.A_{i} / D_{i}\right)$.

We shall study a relationship between those lemmas in the next section.

Received June 28, 1985. 
LEMMA 3. Let $R$ be a left serial algebra with $(A)$, and $e J^{i}=\sum_{j=1}^{n_{i}} \oplus A_{i j}$ with $A_{i j}$ hollow (from Lemma 1). Then $\bar{A}_{i j} \neq \bar{A}_{i j^{\prime}}$ for $j \neq j^{\prime}$, where $\bar{A}_{i j}=A_{i j} / A_{i j} J$.

Proof. Assume $\bar{A}_{i 1} \approx \bar{A}_{i 2} \approx f R / f J: f$ is a primitive idempotent. Then $A_{i j}=a_{i j} R$; $a_{i j} f=a_{i j}(j=1,2)$. Since $R f$ is uniserial, there exists $x$ in $e R e$ such that $a_{i 1}=x a_{i 2}$ (or $\left.a_{i 2}=x a_{i 1}\right)$. If $x \in e J e, a_{i 1} \in e J^{i+1}$. Hence $x \notin e J e$, and $x=e k+j ; k \in K, j \in e J e$ from (A). $a_{i 1}=$ $(e k+j) a_{i 2}=e k a_{i 2}+j a_{i 2} \equiv a_{i 2} k\left(\bmod e J^{i+1}\right)$, contradiction.

THEOREM 1. Assume that $R$ is a left serial algebra with $(A)$. Then the following are equivalent:

1) $R$ is of right local type.

2) $R$ satisfies $(*, 2)$ and $\left|e J / e J^{2}\right| \leqslant 2$ for each $e$.

3) $R$ satisfies $(*, 3)$.

Proof. This is clear from Lemma 3, [3], Theorem 7 and [5].

THEOREM 2. Let $R$ be an algebra over a field. Assume that $R$ is a left serial algebra. Then the following are equivalent:

1) $R$ is of right local type.

2) $R$ satisfies $(*, 3)$.

Proof. This is clear from [1], Theorem 1, [2], Remark 2 and [5].

We give an example for Theorem 1, 2.

$$
R=\left(\begin{array}{cccc}
K & K & \cdots & K \\
& K & & \\
0 & & \ddots & 0 \\
& & & K
\end{array}\right)
$$

is a left serial algebra with $(*, 2)$ and $\left|e J / e J^{2}\right|=n$.

§2. $(*, 1)$

We study, in this section, some left serial rings satisfying $(*, 1)$. First we give

THEOREM 3. Let $R$ be a left serial ring. Then $R$ satisfies $(*, 1)$ if eJ is a direct sum of uniserial modules for each primitive idempotent $e$.

PROOF. Let $C_{i} \supset D_{i}$ be submodules of $A_{i}$ such that $C_{i} / D_{i}$ is simple and $\bar{h}: C_{1} / D_{1} \approx$ $C_{2} / D_{2}$. Since $C_{i}$ is hollow, $C_{1}=x_{1} R$ and $C_{2}=h\left(x_{1}\right) R$, where $h\left(x_{1}\right)$ is a representation of $\bar{h}\left(x_{1}\right)$. We may assume that $x_{1} f=x_{1}$ and $h\left(x_{1}\right) f=h\left(x_{1}\right)$ for a primitive idempotent $f$, since $C_{i}$ is hollow. $R f$ being uniserial, there exists $y$ in $R$ such that $x_{1}=y h\left(x_{1}\right)$ or $h\left(x_{1}\right)=y x_{1}$. Since $\bar{h}$ is 
an isomorphism, we may assume $h\left(x_{1}\right)=y x_{1}$, and $y \in e R e$. For any element $d$ in $D_{1}, d=x_{1} r$; $r \in R$. Then $y d=y x_{1} r=h\left(x_{1}\right) r \in C_{2}$. Hence $h\left(x_{1}\right) r+D_{2}=\bar{h}\left(x_{1}\right) r+D_{2}=\bar{h}\left(x_{1} r\right)+D_{2}=$ $\bar{h}(d)+D_{2}=D_{2}$, and so $y d \in D_{2}$. Let $p_{2}: e J \rightarrow A_{2}$ be the projection. Then $g=P_{2} y_{l} \mid A_{1}$ is in $\operatorname{Hom}_{R}\left(A_{1}, A_{2}\right)$ and $g\left(D_{1}\right) \subset P_{2}\left(D_{2}\right)=D_{2}$, where $y_{l}$ is the left-sided multiplication of $y$. Hence $\bar{h}$ is extendible to $g$ in $\operatorname{Hom}_{R}\left(A_{1} / D_{1}, A_{2} / D_{2}\right)$, and so $(*, 1)$ is satisfied by Lemma 2 .

THEOREM 4. Let $R$ be a left serial algebra with $(A)$ and put $J(e R)=\sum_{i=1}^{n(e)} \oplus A_{i}, J\left(A_{i}\right)$ $=\sum_{j=1}^{n_{i}} \oplus B_{i j}$, where the $A_{i}$ and $B_{i j}$ are hollow. Assume that $J^{4}=0$. Then the following are equivalent:

1) $R$ satisfies $(*, 1)$.

2) $e R$ has the following structure: If $\bar{B}_{i j} \approx C_{i^{\prime} j^{\prime}}$, then $B_{i^{\prime} j^{\prime}}$ is unierial, where $\bar{B}_{i j}=B_{i j} / B_{i j} J$ and $C_{i^{\prime} j^{\prime}}$ is a simple submodule in $J\left(B_{i^{\prime} j^{\prime}}\right),\left(i \neq i^{\prime}\right)$.

Proof. Assume that $R$ satisfies $(*, 1)$ and $\bar{B}_{11} \approx C_{21} \subset e J^{3}$. Put $D_{1}^{*}=$ $J\left(B_{11}\right) \oplus B_{12} \oplus \cdots \oplus B_{1 n_{1}}$. Then $f: J\left(A_{1}\right) / D_{1}^{*} \approx \bar{B}_{11} \approx C_{21}$. Assume that $f$ is extended to $g^{\prime} \in$ $\operatorname{Hom}_{R}\left(A_{2}, A_{1} / D_{1}^{*}\right)$. Since $A_{2} J^{2} \supseteq C_{21}, \bar{B}_{11}=f\left(C_{21}\right)=v^{\prime}\left(C_{21}\right) \subseteq\left(A_{1} / D_{1}^{*}\right) J^{2}=0$. Hence $f$ is extendible to $g$ in $\operatorname{Hom}_{R}\left(A_{1} / D_{1}^{*}, A_{2}\right)$ by Lemma 2. Now, since $A_{1} / D_{1}^{*}$ is uniserial and $g\left(\operatorname{Soc}\left(A_{1} / D_{1}^{*}\right)\right)=C_{21}, g$ is a monomorphism, and so $g\left(A_{1} / D_{1}^{*}\right)$ is a uniserial submodule of $J\left(A_{2}\right)$ which contains $C_{21}$, and $\left|g\left(A_{1} / D_{1}^{*}\right)\right|=2$. However $g\left(A_{1} / D_{1}^{*}\right)$ is a direct sum of two simple modules from the structure of $J\left(A_{2}\right) / C_{21}$ and the fact that $g\left(A / D_{1}^{*}\right) / C_{21}$ is simple, provided that $B_{21}$ is not uniserial. Therefore $B_{21}$ is uniserial. Conversely, if 1 ) is satisfied, then $(*, 1)$ is trivially satisfied. Assume that 2$)$ and $C_{i} \supset D_{i}$ are submodules in $A_{i}$ and $h: C_{1} /$ $D_{1} \approx C_{2} / D_{2}$. Since we use Lemma 2, we may assume that $C_{1} \subset e J^{2}$, because, if $C_{1} \not \subset e J^{2}$, $C_{1}=A_{1}$ and $D_{1}=A_{1} J$. Since $C_{i} / D_{i}$ is simple, $C_{i} / D_{i}=\bar{x}_{i} R$ and we may assume $x_{1} f=x_{1}$, where $x_{i}$ is in $C_{i}$ and $f$ is a primitive idempotent. Further from Lemma 3 we may assume that either $x_{1} \notin e J^{3}$ or $x_{2} \notin e J^{3}$, since $e J^{3}$ is semisimple. Let $x_{1}=b_{1}+\cdots b_{n_{1}} \notin e J^{3} ; b_{i} \in B_{1 i}$. Since $x_{1} f=x_{1}$ and $x_{1}=b_{1} f+\cdots+b_{n_{1}} f, b_{i}=b_{i} f$ and there exists $k$ say 1 from Lemma 3 such that

$$
\left.b_{j} f \in e J^{3} \text { for } j \neq 1 \text { (actually } b_{j} f=0 \text { except one } j^{\prime}\right) \text {. }
$$

Let $x_{2}^{\prime}$ be a representation of $h\left(\bar{x}_{1}\right)$ such that $x_{2}^{\prime} f=x_{2}^{\prime}$. Put $x_{2}^{\prime}=b_{1}^{\prime}+\cdots+b_{n_{2}}^{\prime} ; b_{i}^{\prime} \in B_{2 i}$. If some $b_{i}^{\prime}$ is in $B_{2 i}-J\left(B_{21}\right), \bar{B}_{11}=B_{11} / J\left(B_{11}\right)=\bar{b}_{1} R \approx \bar{b}_{i}^{\prime} R=\bar{B}_{2 i}$, which is a contradiction by Lemma 3 . Hence $b_{i}^{\prime} \in J\left(B_{2 i}\right)$ for all $i$, and so $x_{2}^{\prime}=b_{t}^{\prime}$ for some $t$ by Lemma 3 (cf. (\#)). Then $B_{2 t}$ is uniserial from the assumption. Now $C_{2}=x_{2}^{\prime} R \oplus D_{2}$, since $x_{2}^{\prime} R=\bar{x}_{2}^{\prime} R$ is a simple submodule of $C_{2}$ and there exists $d$ in eJe such that $x_{2}^{\prime}=d x_{1}$. Being $d$ in eJe, $d A_{1} \subset e J^{2}$. By $p_{2}$ we denote the projection of $e J^{2}$ to $B_{2 t}$ and put $g=p_{2} d_{l} \mid A_{1} \in \operatorname{Hom}_{R}\left(A_{1}, A_{2}\right)$. We shall show $g\left(D_{1}\right)=0$. Assume contrarily $g\left(D_{1}\right) \neq 0$. Take an element $z$ in $D_{1}$ such that $g(z) \neq 0 ; z=b_{1}^{\prime \prime}+\cdots+b_{n_{1}}^{\prime \prime}$; $b_{i}^{\prime \prime} \in B_{1 i}$. If $b_{1}^{\prime \prime} \in e J^{3}, 0 \neq p_{2} d z=p_{2}\left(d b_{2}^{\prime \prime}+\cdots+d b_{n_{1}}^{\prime \prime}\right)$ implies that, for some $j, 0 \neq p_{2} d b_{j}^{\prime \prime} \in$ $\operatorname{Soc}\left(B_{2 t}\right)=x_{2}^{\prime} R(j \geqslant 2)$, since $B_{2 t}$ is uniserial. Further $b_{j}^{\prime \prime} \notin J\left(B_{1 j}\right) \subset e J^{3}$ for $p_{2} d b_{j}^{\prime \prime} \neq 0$. Hence $B_{1 j} / B_{1 j} J \approx x_{2}^{\prime} R$, and so $B_{11} / B_{11} J \approx B_{1 j} / B_{1 j} J$, a contradiction. Accordingly, being 
$b_{1}^{\prime \prime} R=B_{11}\left(b_{1}^{\prime \prime} \notin e J^{3}\right)$, there exists $r$ in $R$ such that $b_{1}=b_{1}^{\prime \prime} r$. Put $x_{1}^{\prime}=x_{1}-z r=b_{2}^{\prime \prime}+\cdots+b_{n_{1}}^{\prime \prime}$ $\left(\in C_{1}\right)$. Then $\bar{x}_{1}^{\prime}$ is a generator of $C_{1} / D_{1}$. Further $x_{1}^{\prime} f=b_{2}^{\prime \prime} f^{\prime} f+\cdots+b_{n_{1}}^{\prime \prime} f$ is in $e J^{3}$ from (\#). Hence $x_{1}^{\prime} f R$ is a semisimple submodule of $A_{1}$. $x_{1}^{\prime} f R f \neq 0$ implies that $x_{1}^{\prime} f R$ contains a simple submodule isomorphic to $x_{2}^{\prime} R$, a contradiction. Therefore $g\left(D_{1}\right)=0$, and so $g$ induces an element in $\operatorname{Hom}_{R}\left(A_{1} / D_{1}, A_{2} / D_{2}\right)$, which is an extension of $h$.

COROLLARY. Let $R$ be a left serial algebra with $(A)$. If $J^{3}=0$, then $(*, 1)$ is satisfied.

Finally we give a left serial algebra with $J^{4}=0$ but $(*, 1)$ is not satisfied.

Let $R$ be a vector space over $K$ with basis $\left\{e_{1},(1,2),(1,2)(2,3) \cdots\right\}$ given in the below, we define the product among the basis, $e_{i} e_{j}=e_{i} \delta_{i j}, e_{i}(k, s) e_{j}=(k, s) \delta_{i k} \delta_{s j}$ and products of any four elements $(k, s)$ are zero. Then $R$ is a left serial ring with $J^{4}=0$ and (A).

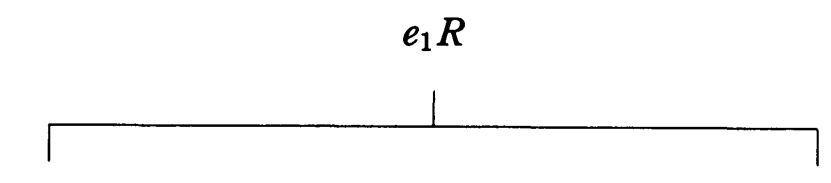

\section{$(1,2) R$}

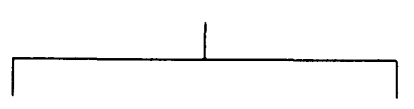

$(1,2)(2,3) R$

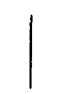

$(1,2)(2,3)(3,5) R$

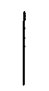

$(1,1) R$

$(1,2)(2,4) R$

$(1,1)(1,2) R$

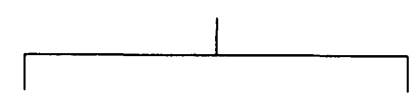

$(1,1)(1,2)(2,3) R \quad(1,1)(1,2)(2,4) R$

$e_{3} R$

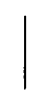

$(2,3) R$

$(2,4) R$

$(3,5) R$

$(2,3)(3,5) R$

$(2,4)(4,6) R$

$\begin{array}{ccc}e_{4} R & e_{5} R & e_{6} R \\ \mid & \end{array}$

$(4,6) R$

Put $A_{1}=(1,2) R, \quad D_{1}=(1,2)(2,4) K \oplus(1,2)(2,3)(3,5) K \oplus(1,2)(2,4)(4,6) K$, $C_{1}=(1,2)(2,3) K \oplus D_{1} . C_{2}=(1,1)(1,2)(2,3) K$ and $D_{2}=0$. Then $h: C_{1} / D_{1} \approx C_{2}$. However $B_{2}=(1,1)(1,2) R$ is not uniserial. Hence $R$ does not satisfy $(*, 1)$. 


\section{References}

[1] Harada, M., On maximal submodules of a finite direct sum of hollow modules III, Osaka J. Math. 22 (1985) 81-98.

[2] Asashiba, H. and Harada, M., ... V, to appear.

[3] Harada, M., Generalizations of Nakayama ring III, to appear.

[4] Sumioka, T., Tachikawa's theorem on algebras of left colocal type, Osaka J. Math. 21 (1984) 624-648.

[5] Tachikawa, H., On rings for which every indecomposable right module has a unique maximal submodule, Math (1959) 200-222.

Department of Mathematics

Osaka City University

Sugimoto 3, Sumiyoshi-Ku

Osaka 558

Japan 